\title{
Thermoelectric properties of the degenerate Hubbard model
}

\author{
V.S. Oudovenko* and G. Kotliar \\ Serin Physics Laboratory, Rutgers University, 136 Frelinghuysen Road, Piscataway, New Jersey 08854, USA
}

(November 12, 2018)

\begin{abstract}
We investigate the thermoelectric properties of a system near a pressure driven Mott-Hubbard transition. The dependence of the thermopower and the figure of merit on pressure and temperature within a degenerate Hubbard model for integer filling $n=1$ is calculated using dynamical mean field theory (DMFT). Quantum Monte Carlo method is used to solve the impurity model. Obtained results can qualitatively explain thermoelectric properties of various strongly correlated materials.
\end{abstract}

72.15.Jf,75.20.Hr,71.10.Fd

The discovery new strongly correlated materials and improvement of theoretical methods offer new perspectives in the search for systems with good thermoelectric performance [1]. The dimensionless figure of merit, $Z T=S^{2} \sigma T / \kappa$, provides us with a quantified measure of the thermoelectric performance of materials. The higher values of the thermoelectric figure of merit corresponds to the better thermoelectric properties. To get maximal thermoelectric response at a fixed temperature, $T$, we need to have maximum possible thermopower, $S$, and the electrical conductivity, $\sigma$, while the thermal conductivity, $\kappa$, should be the smallest possible. The thermal conductivity has two contributions electronic, $\kappa_{e}$, and lattice, $\kappa_{L}$, one, $\kappa=\kappa_{e}+\kappa_{L}$. For some times, the highest value of the figure of merit was equal to one and only a little improvement in getting materials with higher figure of merit has been achieved in the last two decades. Since the electronic structure of strongly correlated electron systems exhibit properties which have no analogy with those of weakly correlated compounds, it is important to understand their physical properties and how they impact the thermoelectric power. Strongly correlated electrons are sensitive to small changes in their control parameters such as temperature, doping and pressure and hence require a detailed investigation. There is now a strong interest in understanding how this changes affect the thermoelectric properties in order to find more efficient material with higher thermoelectric response. Even a modest increase in $Z T$ could substantially impact a number of applications [1].

Two important parameters in strongly correlated electron systems are the carrier concentration and the ratio of the on-site interaction $U$ to the bandwidth $W$. This ratio can be altered by applying external pressure or internal pressure by means of isovalent substitutions.

In this paper we study behaviour of the figure of merit and the thermopower near a pressure driven MetalInsulator Transition (MIT) within framework of the degenerate Hubbard model. Our focus is on the effects of orbital degeneracy. There are several motivations for our study. First, in many strongly correlated materials the orbitals, which bring a major contribution into unusual physical properties, are degenerate. Second, the orbital degeneracy allows us to study the effects of particle hole asymmetry in the Mott insulating state with filling $n=1$ in a natural way (one can also introduce particle hole asymmetry in this case by adding hopping integrals beyond nearest neighbors). Third, the degeneracy will allow us to use the integer filling $n=1$ what in the real system corresponds the situation when the number of the electrons is equal to the number of sites. We use Coulomb repulsion $U$ and temperature $T$ as the parameters to vary and take the half bandwidth $D$ to be unity.

While the calculation of the numerical value of the thermoelectric coefficient requires the detailed modeling of the band structure and interaction constants of the relevant compounds, we expect that the qualitative features related to the thermoelectricity of a Mott insulator at integer filling can be captured by the simplest model, the degenerate Hubbard model, and we undertake this study in the paper. Indeed the trends discussed here, are found in $\mathrm{NiS}_{2-\mathrm{x}} \mathrm{Se}_{\mathrm{x}}$ and $\mathrm{Ni}_{1-\mathrm{x}} \mathrm{Co}_{\mathrm{x}} \mathrm{S}_{2}$ as we discuss toward the end of this paper. The algorithms necessary for more realistic calculations of thermoelectricity will be discussed in a future publication [2].

To treat the two-band degenerate Hubbard model we use the Dynamical Mean Field Theory (DMFT) [3]. Recent development of the DMFT has given a boost to the study of strongly correlated systems and their properties. In particular it has resulted in a detailed understanding of the Mott transition.

Previous studies of thermoelectric properties using DMFT were done within framework of the one-band Hubbard model [4] [- strong temperature and doping dependence of the thermopower was reported. Pálsson and Kotliar considered the orbital degeneracy away from half filling in the limit of infinite interaction strength [7. A good description of the Seebeck coefficient dependence on temperature and doping for titanates was obtained.

In this paper we focus on the integer occupancy case with orbital degeneracy. We also keep $U$ finite and study dependence of thermoelectric properties on the interaction. Changing the ratio of the interaction strength one can mimic the effects of pressure.

The $N$-fold degenerate Hubbard Hamiltonian reads: 
$H=-\sum_{\langle i j\rangle, \sigma} t_{i j} c_{i \sigma}^{\dagger} c_{j \sigma}+\frac{U}{2} \sum_{i, \sigma \neq \sigma^{\prime}} n_{i \sigma} n_{i \sigma^{\prime}}-\mu \sum_{i, \sigma} n_{i \sigma}$,

where $\langle i j\rangle$ runs over nearest neighbor sites, $\sigma$ is the spin and orbital index which runs from 1 to $N$. The twoband degenerate Hubbard model corresponds to $N=4$. The hopping matrix is given by $t_{i j}, U$ is the Coulomb repulsion and $\mu$ is the chemical potential. We also want to restrict ourselves to the paramagnetic phase (in both spin and orbit indices) i.e. we are interesting in metalinsulator transition and its influence on transport properties due to electronic correlations only. It is worth to mention that orbital degeneracy suppresses the magnetic correlations enough to make our assumption easily fulfilled [8]. To make computations easier further we assume $t_{i j}=t$.

In this paper we use the model on a cubic lattice. For calculation of thermodynamic properties we use the semicircular density of states (DOS) $\rho(\epsilon)=$ $(2 / \pi D) \sqrt{1-(\epsilon / D)^{2}}$, with the half-bandwidth $D=$ $W / 2=2 t$. The semicircular DOS corresponds to an infinite coordination Bethe lattice which, as it was found earlier 3, gives a good description of three dimensional systems.

The next step is to solve the Hubbard model. The standard way in DMFT to do it is to map the lattice Hubbard model onto the effective impurity problem, which is a generalized single impurity Anderson model, where the operators carry an orbital index. Supplemented with the self-consistency condition [3] solution of the impurity model gives us the solution of the original Hubbard model. Quantum Monte Carlo method with extended Hirsch-Fye algorithm [9,10] is used as the impurity solver. To reduce computational errors (the Trotter breakup) imaginary time interval is set to $\Delta \tau=1 / 4$. During the self-consistency procedure one needs to make direct and inverse Fourier transformations for the Green's functions (GF). In the present calculations we use a modified Fourier transformation (FT) in order to get correct results (for details see Appendix).

The output of the self-consistent procedure described above is the Green's function on imaginary time axis. To calculate the thermoelectric properties we need to know behaviour of GF on real frequency axis. The Maximum Entropy (ME) method is used to make the analytical continuation of imaginary-time GF to DOS on the real frequency axis. Knowing imaginary part of GF we reconstruct frequency dependence of the real part $G(\omega)$ through Kramers-Kronig relations. Obtained GF is used to calculate the transport coefficients.

Using Kubo formalism one can express thermoelectric coefficients in terms of current-current correlation functions which are reduced within DMFT to averages over the spectral density function $\rho(\epsilon, \omega)[7,11]$ :

$$
S=-\frac{A_{1}}{e A_{0}}, \quad \sigma=\frac{e^{2}}{T} A_{0}, \quad \kappa=\left(A_{2}-\frac{A_{1}^{2}}{A_{0}}\right),
$$

where the coefficients $A_{n}$ have the following form,

$$
\begin{aligned}
A_{n} & =\frac{1}{V} \sum_{k, s} \int d \omega \rho_{s}^{2}(k, \omega)\left(\frac{\partial \epsilon_{k}}{\partial k_{x}}\right)^{2}\left(-T \frac{\partial f(\omega)}{\partial \omega}\right)(\beta \omega)^{n} \\
& =N_{d e g} \pi \int_{-\infty}^{\infty} d \omega d \varepsilon \frac{\rho^{2}(\varepsilon, \omega)(\omega \beta)^{n}}{4 \cosh ^{2}\left(\frac{\beta \omega}{2}\right)} \Phi(\varepsilon)
\end{aligned}
$$

where $T$ is temperature, $f(\omega)$ is the Fermi distribution function, $s$ describes spin and orbital indexes which runs from 1 to $N_{\text {deg }}$. The spectral density, $\rho_{s}(k, \omega)$, and the transport function, $\Phi(\varepsilon)$, contain the relevant information about the bare band structure, $\epsilon_{k}$ :

$$
\Phi(\varepsilon)=\frac{1}{V} \sum_{k}\left(\frac{\partial \epsilon_{k}}{\partial k_{x}}\right)^{2} \delta\left(\varepsilon-\epsilon_{k}\right) .
$$

As it is seen from the above formulae (3) and (4) contribution to the thermopower strongly depends on temperature. For low temperatures only states close to Fermi surface (FS) contribute to thermoelectric properties while for high temperatures the entire Brillouin zone is important. Coulomb repulsion, $U$, acts on the transport coefficients via changes in the spectral density function, $\rho(\varepsilon, \omega)$. There is a very simple mnemonic rule to define the sign of the thermopower. For small temperatures it depends on DOS slope at the Fermi energy: if the DOS curve rises when it crosses the Fermi energy then the sign is negative and vice versa. For very high temperatures it depends on weight of the Hubbard bands: if weight of the Hubbard band above the Fermi energy is larger than contribution from the band below the Fermi level then the thermopower is negative. This rule will help us easily understand the thermopower behaviour analyzing temperature dependence of DOS.

In Fig. 11 we plot dependence of the thermopower on interaction strength $U$. The thermopower changes sign in a region of Coulomb repulsion $2.5<U<3$, the region where the system undergoes metal-insulator transition at low temperatures. The MIT for high temperatures transforms into the metal-insulator crossover what is reflected in the spectral function (DOS) behaviour presented in Fig. 2. It is worth to notice quite strong $S$ dependence on $U$ in that crossover region. When $U \rightarrow 0$ the thermopower will have a finite value depending on temperature and in opposite limit $U \rightarrow \infty$ we expect saturation of the thermopower dependence which will be also temperature dependent. The interaction dependence of the thermopower helps us qualitatively understand the pressure influence onto the system as well as behaviour of a system (e.g. $\mathrm{NiS}_{2-\mathrm{x}} \mathrm{Se}_{\mathrm{x}}$ ) changing its properties from insulating to metallic ones by varying the bandwidth. It follows from the fact that the only important parameter in the system is the the ratio $U / W$ and one can vary any 
of two variables to get qualitative description of a system properties.

Behaviour of the figure of merit on interaction strength (Fig. 3) is possible to understand from its definition. It has minimum where the thermopower changes sign. After the sign change it substantially increases and has maximum for higher values of $U$. Rather high values of $Z T$ (more than one) can be explained by the absence of the lattice contribution in the thermal conductivity. If one takes into account the lattice thermal conductivity the figure of merit drops to value below one. Usual values of lattice contribution into the thermal conductivity in transition metal-oxides lay in region $0.1-10 \mathrm{~W} / \mathrm{mK}$. We believe that $1 \mathrm{~W} / \mathrm{mK}$ is a reasonable value for pyrites. We see that the figure of merit is maximal for interaction $\mathrm{U}$ between 4 and 4.5 , but even in this region obtained figure of merit could not be competitive with the one for semiconductors due to the lattice contribution into the thermal conductivity.

Temperature dependence of the thermopower for $U=$ 3 presented on Fig. 4 can be easily explained from analysis of DOS temperature dependence in Fig. 5. For very low temperatures the thermopower has linear dependence (one can show it analytically, it is nearly impossible at the present stage to reach very low temperatures with QMC) and negative sign indicating presence of quasiparticles in the system what is clearly seen in Fig. 5 $(\beta=16)$ where a good quasiparticle peak at the Fermi energy is observable. With increasing temperature a smooth crossover from quasiparticles excitations to incoherent ones reflected in non-monotonic thermopower dependence which changes sign from negative to positive one. In Fig. 5 for temperatures $\beta=12$ and $\beta=8$ we see how quasiparticle peak of DOS gets lower and then disappears. From this observation we can conclude that positive value of $S$ tells us that the band structure consists from two Hubbard subbands only. Similar behaviour of the thermopower (with the sign change) occurs in the single band Hubbard model upon doping [11 and in the Hubbard model with frustration [6] as well as in the periodic Anderson model [12]. Further temperature behaviour of the thermopower is new one. At temperature $\beta=8$ in Fig. A the thermopower reaches its maximum and then starts decreasing (we should notice here that temperature $\beta=4$ is the coherent temperature for the studied system). For $\beta=4$ the spectral function consists from two bands, where lower Hubbard band, which is closer to the Fermi energy has greater contribution into the thermopower than the upper one, which is located a bit further from the chemical potential position. With increasing temperature two bands became less asymmetric to the Fermi energy and the weight from the upper Hubbard band $(n=1)$ is larger than the contribution from the lower band. It means that the thermopower should became negative as it seen in Fig. 4 (the last point). And finally, for very high temperature the two bands collapse into the one. But the sign of the thermopower remains unchanged by the same reason as we discussed above. The thermopower sign change in the high temperature limit due to Mott-Hubbard bands collapse was reported in 13 .

To gain further understanding of the temperature and interaction dependence of the thermopower we study its high-frequency behaviour in the high-temperature limit. To do so we generalize the thermoelectric response to finite frequencies (ac-thermopower) [14]:

$$
S(\omega)=-\frac{1}{e T} \frac{L^{12}(\omega)}{L^{11}(\omega)}
$$

where coefficients $L^{11}$ and $L^{12}$ are defined as

$$
L_{j i}^{1 n}(\omega)=\frac{i e^{\beta \Omega}}{\omega \beta} \sum_{\mu \nu} \frac{\left\langle\mu\left|q_{i}\right| \nu\right\rangle\left\langle\nu\left|J_{j}^{n}\right| \mu\right\rangle\left(e^{-\beta \varepsilon_{\mu}}-e^{-\beta \varepsilon_{\nu}}\right)}{\omega+\varepsilon_{\mu}-\varepsilon_{\nu}+i \delta} .
$$

Here $e^{-\beta \Omega}=\operatorname{Tr}\left(e^{-\beta(H-\mu N)}\right), i, j=x, J_{x}^{1}=j_{x}$, $J_{x}^{2}=j_{x}^{Q}, j_{x}$ and $j_{x}^{Q}$ are electrical and heat currents in $x$ direction correspondingly, $q_{x}$ is the polarization operator satisfying $j_{x}(t)=\partial q_{x}(t) / \partial t$.

Expanding numerator and denominator of the thermopower $\left(L^{11}(\omega)\right.$ and $\left.L^{12}(\omega)\right)$ in frequency and dividing one onto another we obtain the following expansion of the thermopower in the high-frequency limit:

$$
S(\omega)=-\frac{\beta}{e}\left(\frac{\left\langle\left[q_{x}, j_{x}^{Q}\right]\right\rangle}{\left\langle\left[q_{x}, j_{x}\right]\right\rangle}+\mathcal{O}(\omega)\right)
$$

The relevant commutators are given by:

$$
\begin{aligned}
& -\frac{1}{i t}\left[q_{x}, j_{x}^{Q}\right]= \\
& \sum_{j s}\left\{t\left[c_{j+2 x}^{\dagger} c_{j s}+c_{j-2 x, s}^{\dagger} c_{j, s}\right]+\mu\left[c_{j+x, s}^{\dagger} c_{j, s}+c_{j-x, s}^{\dagger} c_{j, s}\right]\right. \\
& \left.-U\left[c_{j+x, s}^{\dagger} c_{j, s} \sum_{s^{\prime} \neq s} n_{j+x s^{\prime}}+c_{j-x, s}^{\dagger} c_{j, s} \sum_{s^{\prime} \neq s} n_{j-x} s^{\prime}\right]\right\}
\end{aligned}
$$

This expression consists of three terms. The first one, proportional to $t$, doesn't contribute to the hightemperature expansion if one takes into account only nearest neighbour hoppings. The second term (proportional to chemical potential) similar to what we have in the denominator and the last one proportional to $U$ contribute to high-temperature expansion of the thermopower. The final result we end up with is presented below

$$
S(\omega) \stackrel{T \rightarrow \infty}{\longrightarrow}-\frac{\beta}{e}\left(-\mu+U \frac{\bar{n}}{1-n}\right)
$$

where $\bar{n}$ corresponds to contribution to filling $n$ coming from $N_{\text {deg }}-1$ degrees of freedom (spin and orbital) and is equal to $\sum_{\sigma, \sigma^{\prime} \neq \sigma}\left\langle\left(1-n_{\sigma}\right) n_{\sigma^{\prime}}\right\rangle$. If one assumes hightemperature behaviour of the chemical potential in the 
form $\mu / T=\alpha$, where $\alpha$ should be negative for the filling $n<\frac{1}{2} N_{\text {deg }}$, one obtains that the sign of the thermopower should be negative in the high-temperature limit. Hence ac- and dc- thermopowers have the same sign in the hightemperature limit.

It is quite difficult to make a direct comparison of a model calculation and situation in real materials due to complexity and wide variety of real structures. To make a comparison with our model calculations we need a material with degenerate $e_{g}$ band hosting one electron (hole). As we mentioned in the introduction a good candidates for the comparison are pyrites compounds with doubly degenerate $3 d-e_{g} \mathrm{Ni}$ band. An attempt to understand high-temperature dependence of the thermopower in this materials on base of one-band electron correlation theory with a good fit of experimental data using six parameters model was given some years ago in work of Kwizera et al. 15. To explain low temperature dependence of the thermopower it was suggested that both holes and electrons participate in charge transport and a two-band model was proposed as an appropriate model for data interpretation [13]. Our calculations show that the twoband degenerate Hubbard model treated within DMFT, is sufficient model for at least qualitative description of pyrites in the whole temperature range [13, 15, 16]. In the case of half-filled $\mathrm{Ni} 3 d-e_{g}$ band in $\mathrm{NiS}_{2-\mathrm{x}} \mathrm{Se}_{\mathrm{x}}$ theoretical model under consideration due to the symmetry in the system should give zero thermopower for all temperatures, while experimentally it is zero only for temperature $T<100 \mathrm{~K}$. For higher temperatures the thermopower becomes positive. It is clear that one needs to take into account effects of other bands (filled $t_{2 g}$ band in the first turn). The thermopower behaviour in $\mathrm{Ni}_{1-x} \mathrm{Co}_{\mathrm{x}} \mathrm{S}_{2}$ with temperature looks rather similar as in $\mathrm{NiS}_{2-x} \mathrm{Se}_{\mathrm{x}}$ but with Co substitution of $\mathrm{Ni}$ atoms occupancy of $3 d-e_{g}$ band changes from two $(x=0)$ to one $(x=1)$. One can track the experimental situation in $\mathrm{NiS}_{2-\mathrm{x}} \mathrm{Se}_{\mathrm{x}}$ analyzing model calculations (for temperatures low enough to neglect contributions coming from other bands) especially in the case $\mathrm{CoS}_{2}$ which corresponds to one-quarter filled $3 d-e_{g}$ band.

In conclusion, we calculated dependencies of the thermopower and the figure of merit on interaction strength and temperature of the thermopower in the two-band degenerate Hubbard model for integer filling $n=1$. The strong dependence on studied parameters was obtained. Analytical estimations of the high-frequency limit of acthermopower is provided. In the high-temperature limit ac- and dc- thermopowers have the same sign (negative for filling $n<0.5$ ). We expect that the thermopower behaviour in the three-band degenerate Hubbard model would behave in a similar way. It means that we can qualitatively understand behaviour of the thermoelectric properties in a wide range of strongly correlated materials where a major role played by $d$ - and $f$-shell electrons.

Systems near the temperature driven Mott transi- tion exhibit very rich thermoelectric behaviour. As we cross the localization-delocalization threshold the thermopower increases substantially. There are the hightemperature precursors of the first order Mott transition that takes place at lower temperature. As the temperature is lowered, and the critical Mott endpoint is approached, the specific heat diverges and the entropy jumps. The figure of merit is low in this crossover regime because of the great deal of cancellation between the quasiparticle contributions which are in our model electron like, and the Hubbard band contributions which are hole like. A detailed phase diagram temperature versus filling for different values of $U$ using the current approach will be studied elsewhere.

The authors are indebted to G. Pálsson, A.I. Lichtenstein for many helpful and stimulating discussions. We also acknowledge usage of Rutgers Computational Grid (RCG) PC cluster as well as NERSC Cray T3E supercomputer which made our computations possible. The research was supported by American chemical society Petroleum Research Fund grant ACS-PRF \# 33495-ACS.

* Permanent address: BLTPh, JINR, 141980 Dubna, Russia.

[1] G. Mahan, B. Sales, and J. Sharp, Physics Today 50, 42 (1997).

[2] G. Pálsson, V.S. Oudovenko and G. Kotliar, (in preparation).

[3] For a recent review, see: A. Georges, G. Kotliar, W. Krauth and M.J. Rozenberg, Rev. Mod. Phys. 68, 13 (1996).

[4] Th. Pruschke, D.L. Cox, and M. Jarrell, Phys. Rev. B 47, 3553 (1993).

[5] M.J. Rozenberg, G. Kotliar, H. Kajueter, G.A. Thomas, D.H. Rapkine, J.M. Honig, and P. Metcalf, Phys. Rev. Lett. 75, 105 (1995).

[6] J. Merino, and R.H. McKenzie, Phys. Rev. B 61, 7996 (2000).

[7] G. Pálsson and G. Kotliar, Phys. Rev. Lett. 80, 4775 (1998).

[8] H. Kajueter and G. Kotliar, Int. J. Mod. Phys. 11, 729 (1997).

[9] J.E. Hirsch and R.M. Fye, Phys. Rev. Lett. 56, 2521 (1986); H.Q. Lin and J.E. Hirsch, Phys. Rev. B 37, 1864 (1988).

[10] K. Takegahara, J. Phys. Soc. Jpn. 62, 1736 (1992).

[11] Th. Pruschke, M. Jarrell and J. Freericks, Adv. Phys. 44, 187 (1995).

[12] H. Schweitzer and G. Czycholl, Phys. Rev. Lett. 67, 3724 (1991).

[13] X. Yao, J.M. Honig, T. Hogan, C. Kannewurf and J. Spalek, Phys. Rev. B 54, 17469 (1996).

[14] G.D. Mahan, Many-particle physics, (Plenum, New York, N.Y., 2nd edition, 1993). 
[15] P. Kwizera, M.S. Dresselhaus, and D. Adler, Phys. Rev. B 21, 2328 (1980).

[16] A.K. Mabatah, E.J. Yoffa, P.C. Eklund, M.S. Dresselhaus, and D. Adler, Phys. Rev. Lett 39, 494 (1977).

[17] M. Potthoff, T. Wegner, W. Nolting, Phys. Rev. B 55, 16132 (1997).

[18] B. Velicky, S. Kirkpatrick, and H. Ehrenreich, Phys. Rev. 175, 747 (1968).

[19] H. Kajueter, Ph.D.Thesis, Rutgers University, 1996.

\section{APPENDIX A: FOURIER TRANSFORMATION IN QMC CALCULATIONS}

In the self-consistent procedure to solve the impurity problem using QMC method [3] we need to do two direct Fourier transformations for GFs $G(\tau)$ and $G_{0}(\tau)$ (the Weiss function) and one inverse Fourier transformation (iFT) for $G_{0}(\tau)$ only. Function $G_{0}(\tau)$ is an input for QMC simulations producing $G(\tau)$. Two direct Fourier transformations are necessary to impose the selfconsistency condition which is usually written in frequency space. Both, GF and the Weiss function contain the discontinuities at $\tau=0$ and $\tau=\beta$. The discontinuities in these functions and their derivatives determine the high-frequency behaviour of their frequency dependent Fourier transformations $G(\omega)$ and $G_{0}(\omega)$. As it is well known that information about high-frequency behaviour of GFs is absent in QMC simulations themselves (maximum frequency available is the Nyquist frequency $\omega_{\max }=1 / 2 \Delta$, where is $\Delta$ is imaginary time interval). The high frequency (small imaginary time) information is not contained in the QMC itself but has to be incorporated into the splining procedure using additional information.

This information is available from calculation of corresponding moments of the GFs. To make this connection clear, we make consecutive integration by parts of the Fourier integral:

$$
\begin{aligned}
G\left(i \omega_{n}\right) & =\sum_{k=0}^{N} \frac{(-1)^{k+1}\left(G^{(k)}(0)+G^{(k)}(\beta)\right)}{\left(i \omega_{n}\right)^{k+1}} \\
& +\frac{(-1)^{N+1}}{\left(i \omega_{n}\right)^{N+1}} \int_{0}^{\beta} \mathrm{e}^{i \omega_{n} \tau} \frac{\partial^{N+1} G(\tau)}{\partial \tau^{N+1}} d \tau .
\end{aligned}
$$

Values of GF sum and it's derivatives can be expressed via corresponding momenta. These momenta can be calculated similar to the one-band Hubbard model [17]. A momentum of $k$ degree is defined as follows:

$$
M^{(k)}=\int_{-\infty}^{+\infty} d \omega \omega^{k} \rho(\omega)
$$

We can bind Eqs. (A1) and A2 writing the following expression for sum of GFs and it's derivatives in imaginary-time space:

$$
G^{(k)}(0)+G^{(k)}(\beta)=M^{(k)},
$$

where $k=0, \ldots N$. To make direct Fourier transformation, first, we interpolate $G(\tau)$ defined in $L$ points $(L$ number of time slices) and then take analytical Fourier transformation of resulting function. The procedure of interpolation plays a key role. We used the cubic spline interpolation for $G(\tau)$ where condition of continuous second derivative $\left(G^{(2)}\right)$ is imposed. Using this condition we write a system of linear equations to find interpolation coefficients. To close the set of linear equations we need to put correct boundary conditions. The standard way to do it is to set the second derivatives at the end points of the time interval $[0, \beta]$ to zero (so called, natural spline). In our approach we use analytical information about the momenta (sum of the second and first derivatives at the end points, $M^{(1)}$ and $\left.M^{(2)}\right)$.

To obtain this information we need to know expansion of the self-energy in $1 / \omega$ series or in another words we need to know first two momenta of the self-energy.

$$
\begin{aligned}
& M_{\Sigma}^{(0)}=(2 N-1) U n, \\
& M_{\Sigma}^{(1)}=(2 N-1) U^{2} n(1-n)+2 U^{2} C_{2}^{2 N-1}\langle n n\rangle,
\end{aligned}
$$

where $N$ is the number of bands and $C_{n}^{k}=\frac{(k) !}{n !(k-n) !}$ is a combinatorial factor which arrives due to the spin and orbital degeneracy.

The self-energy expansion contains density-density correlations functions for different spins and orbitals. One way to obtain them is to use an approximate scheme which is accurate at high frequencies such as the coherent potential approximation (CPA) [18], [19]. Another possibility is to evaluate the correlation functions which enter in Eq. (A4) using the QMC procedure which allows us to compute arbitrary local correlation functions such as the density-density correlators. The self-consistency procedure ensures us that they are correct correlation functions when the self-consistency is reached. Having correct momenta we solve the set of linear equations to obtain the cubic spline function which is Fourier transformed analytically.

The power of modern computers allows us to use in imaginary time $(\tau)$ space up to $L=256$ time slices only, while in frequency $(\omega)$ space we do not have such limitations and can use as much frequency points as we want but only frequencies less than the Nyquist frequency have physical meaning (usually it is less than $2^{8}$ ). As we know GF has $1 / \omega$ asymptotic behaviour and dealing with the finite number of frequency points we introduce the finite energy cut-off. Cutting off the tail of GF, we remove the discontinuity from the Fourier transformed function in $\tau$ space. To correct the situation we subtract the highfrequency tail from GF and make FT of the obtained 
function numerically and make Fourier transform of the tail analytically. Finally, in $\tau$ space we sum up the obtained functions and as the result we have the correct inverse Fourier transformation, we call it the "new" one.

To demonstrate the difference between two "old" and "new" Fourier transformations we calculate the selfenergy in the considered model for $U=4$ and $\beta=8$. Results are plotted in Fig. 6 where the "new" solution is drawn by solid line and the "old" one is plotted by dashed line. We see that the "old" one has the region where the self-energy changes sign ("overshoots"). It corresponds to unphysical contribution to self-energy which should always keep the same sign for positive or negative frequencies.

Finally, we stress that difference between two Fourier transformations becomes substantial especially in critical regions of parameters: low doping, high values of $U$ and low temperatures $T$.
FIG. 1. Dependence of the thermopower, $S$ ( in units of $\left.k_{B} / e=86 \mu V / K\right)$, on Coulomb interaction, $U$ (in $D=1 \mathrm{eV}$ ) for $\beta=8$.

FIG. 2. Spectral functions (DOS) for $U=2.5$ and 3 for $\beta=8$.

FIG. 3. The figure of merit, $Z T$, vs. interaction strength, $U$, for temperature $\beta=8$ and different values of the lattice conductivity $\kappa_{L}[\mathrm{~W} / \mathrm{mK}]$.

FIG. 4. Temperature dependence of thermopower, $S$ for $U=3$. The solid line is guide for the eye.

FIG. 5. Temperature dependence of density of states for $U=3$.

FIG. 6. Dependence of imaginary part of the self-energy on Matsubara frequency axis $\omega(\beta=8)$ for two different Fourier transformations 'old' (dashed line) and 'new' (solid line) one. 


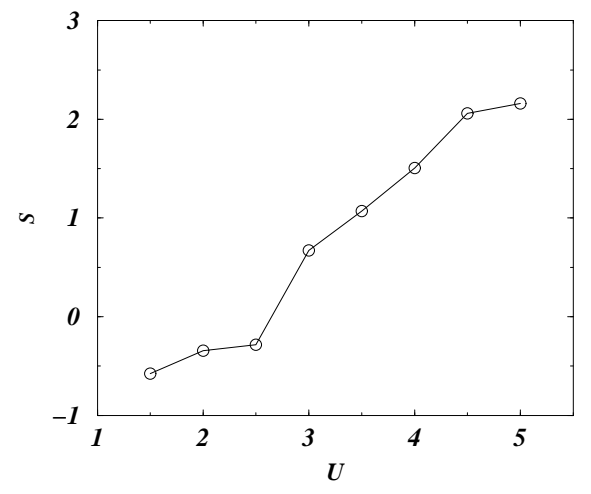

FIG. 1

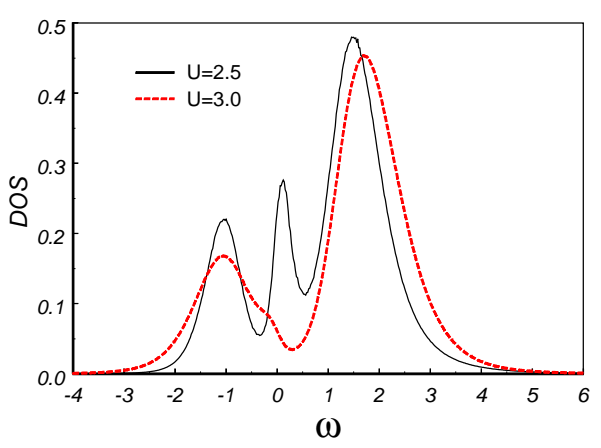

FIG. 2

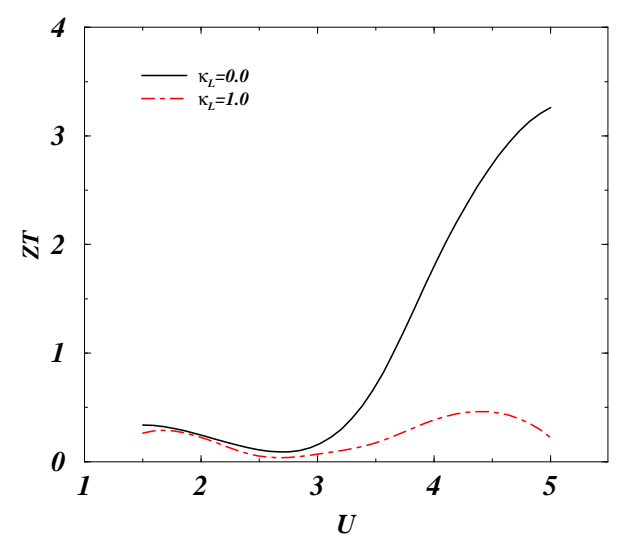

FIG. 3

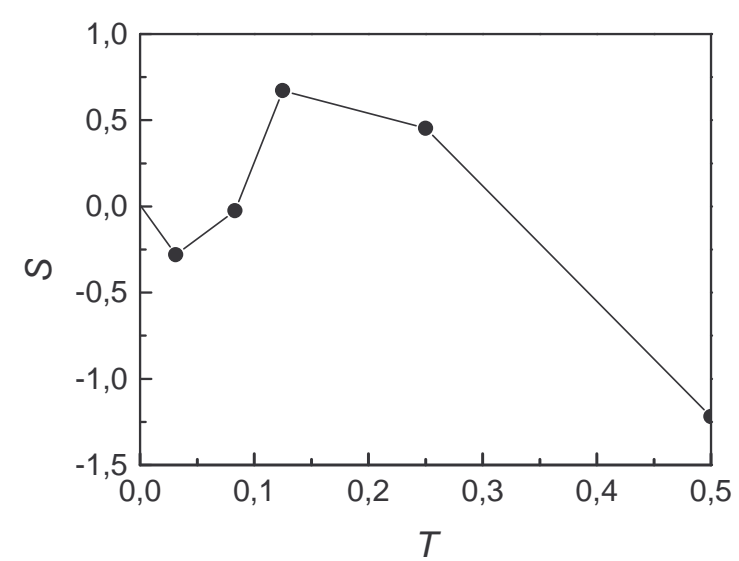

FIG. 4

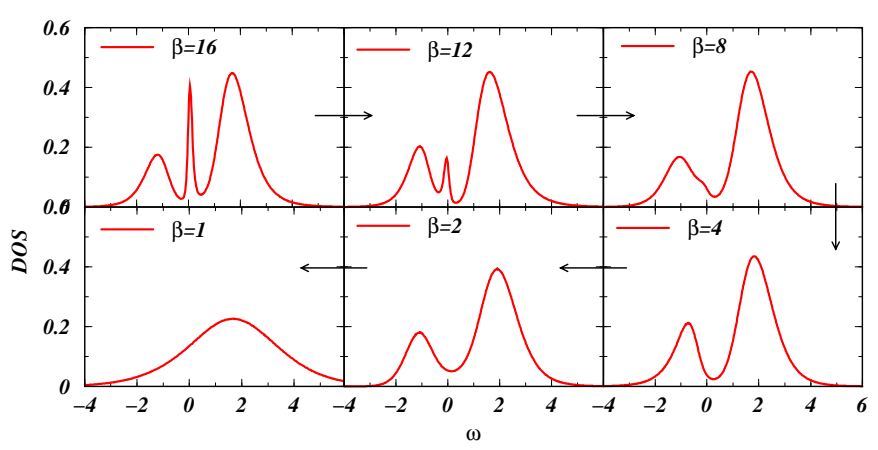

FIG. 5

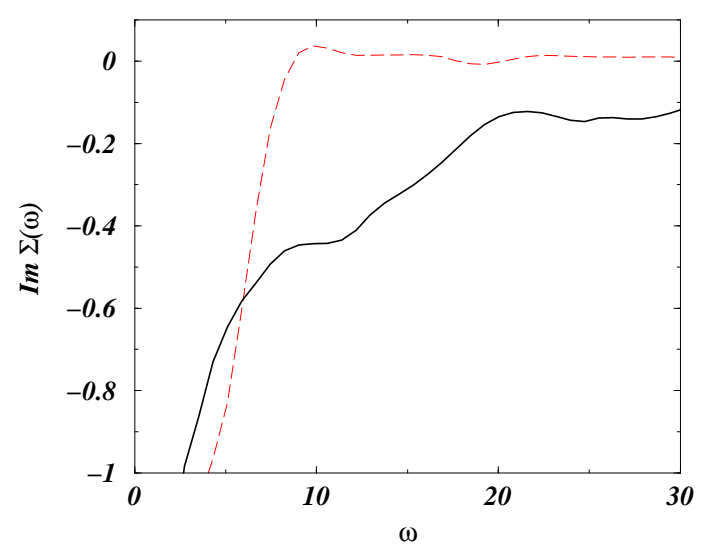

FIG. 6 